\title{
Das intraoperative CT
}

\author{
Christoph-H. Hoffmann, Philipp Wilde, Reinhard Hoffmann, Frank Kandziora
}

\section{Zusammenfassung}

Das intraoperative CT (iCT) ist ein modernes bildgebendes Verfahren in der Wirbelsäulen- und Unfallchirurgie. Eingriffe, die eine hohe Präzision erfordern, sind für den Einsatz der spinalen Navigation auf Grundlage intraoperativer CT-Bildgebung besonders geeignet. Hierzu zählen die korrekte transpedikuläre Schraubenplatzierung bei schmalen Pedikeln, intraossäre Tumoren, ausgeprägte Deformitäten, Revisionseingriffe und myelonnahe bzw. intramedulläre Operationen im Bereich der Wirbelsäulenchirurgie. Unfallchirurgische Indikationen bestehen v. a. bei Beckeneingriffen sowie bei der operativen Versorgung komplexer Gelenkverletzungen. Das intraoperative CT ermöglicht eine zeitnahe Kontrolle des Operationsergebnisses mit einer höheren Bildqualität sowie einem wesentlich größeren Field of View im Vergleich zur intraoperativen 3D-Fluoroskopie. Die Strahlenbelastung ist für den Patienten höher als beim fluoroskopischen Verfahren, für den Operateur jedoch geringer. Die Kosteneffektivität des iCTs sowie der iCT-gestützten Navigation ist aufgrund der noch immer hohen Investitionskosten niedrig, unter medizinischen Aspekten ist das iCT-gestützte Navigieren jedoch gegenüber anderen Monitoringverfahren klinisch oftmals vorteilhaft.

\section{Intraoperative CT}

Intraoperative CT (iCT) is an up-todate adjunct to spinal and trauma surgery. Any procedure requiring high precision is especially amenable for spinal navigation with iCT scanning. Indications in the field of spinal surgery include placement of transpedicular screws in small thoracic or cervical pedicles, intraosseous tumours, high-grade deformities, revision surgeries, intramedullary procedures and surgeries in close proximity to the spinal cord. In addition, iCT scanning may offer a benefit in the treatment of pelvic and complex joint injuries. Moreover, iCT scanning allows for shortterm validation of surgical results with a higher imaging quality and larger field of view compared to 3D-fluoroscopy. Radiation exposure for patients is higher compared to conventional fluoroscopy, but it is lower for the surgeon. Cost effectiveness is low due to relatively high investment costs, however, when comparing iCT navigation with other monitoring procedures, it is advantageous from the medical point of view.

\section{Entwicklung}

Intraoperative CT-Untersuchungen wurden bereits in den 1970er-Jahren bei stereotaktischen Operationen durchgeführt, um Zielpunkte exakt zu definie-

OP-JOURNAL 2014; 30: 150-158

(c) Georg Thieme Verlag KG Stuttgart · New York DOI http://dx.doi.org/10.1055/s-0034-1382991

ren. Dabei bildete der stereotaktische Rahmen die Referenzierungsbasis. Aus dem CT ließen sich die Vektoren bzw. Koordinaten definieren, welche den linearen Weg durch den Schädel zum Zielpunkt vorgaben. Die Einführung der Neuronavigation mit der Möglichkeit, auf der Basis eines präoperativ durchgeführten CTs ohne stereotaktischen Rahmen ein beliebiges Ziel kurzfristig auch auf einem nicht-linearen Weg an- zufahren, führte letztlich zur iCT-Untersuchung als Echtzeit-Bildgebung mit Darstellung des intraoperativen Situs.

Die Domäne der iCT-Bildgebung liegt heute im Bereich der spinalen und traumatologischen Chirurgie, aber auch in anderen Bereichen wie z.B. der Schädelbasischirurgie, überall dort, wo es auf die exakte Darstellung insbesondere knöcherner Strukturen ankommt. Durch eine intraoperative CT-Angiografie kann die Darstellung vaskulärer Strukturen bei besonderen Fragestellungen ergänzt werden. Das iCT ist dabei integrativer Bestandteil der Navigation, kann aber auch als unmittelbare postoperative Kontrolle eingesetzt werden.

\section{Präzision}

Die gute räumliche Auflösung ossärer Strukturen bildet die Basis für die intraoperative Bildgebung sowie die hohe Genauigkeit der Navigation. Neben dem technisch fassbaren Registrierungsfehler und der intraoperativ durchgeführten Plausibilitätskontrolle lassen sich post hoc radiologische Parameter als Maß für die Präzision heranziehen. Laine et al. [1] untersuchten in einer prospektiven randomisierten Studie die Lage lumbaler Pedikelschrauben in einer Serie von 100 Patienten. Dabei verglichen sie die Lage der entweder konventionell mit intraoperativer Durchleuchtungskontrolle oder navigiert unter Verwendung eines präoperativ durchgeführten CTs eingebrachten Schrauben durch eine postoperative CT-Kontrolle anhand einer Graduierung, die das Ausmaß der Pedikelperforation (extrapedikuläre Lage: offset in $\mathrm{mm}$ ) und deren Richtung (medial, lateral, inferior, superior) berücksichtigt. In der Gruppe der konventionell eingebrachten Pedikelschrauben lag die Pedikelperforationsrate bei $13,4 \%$, in der navigierten Gruppe bei $4,6 \%(p=0,006)$. Perforationen über $4 \mathrm{~mm}$ traten in der konventionellen Gruppe in 1,4\%, in der navigierten Gruppe in keinem Fall auf. Zahlreiche 


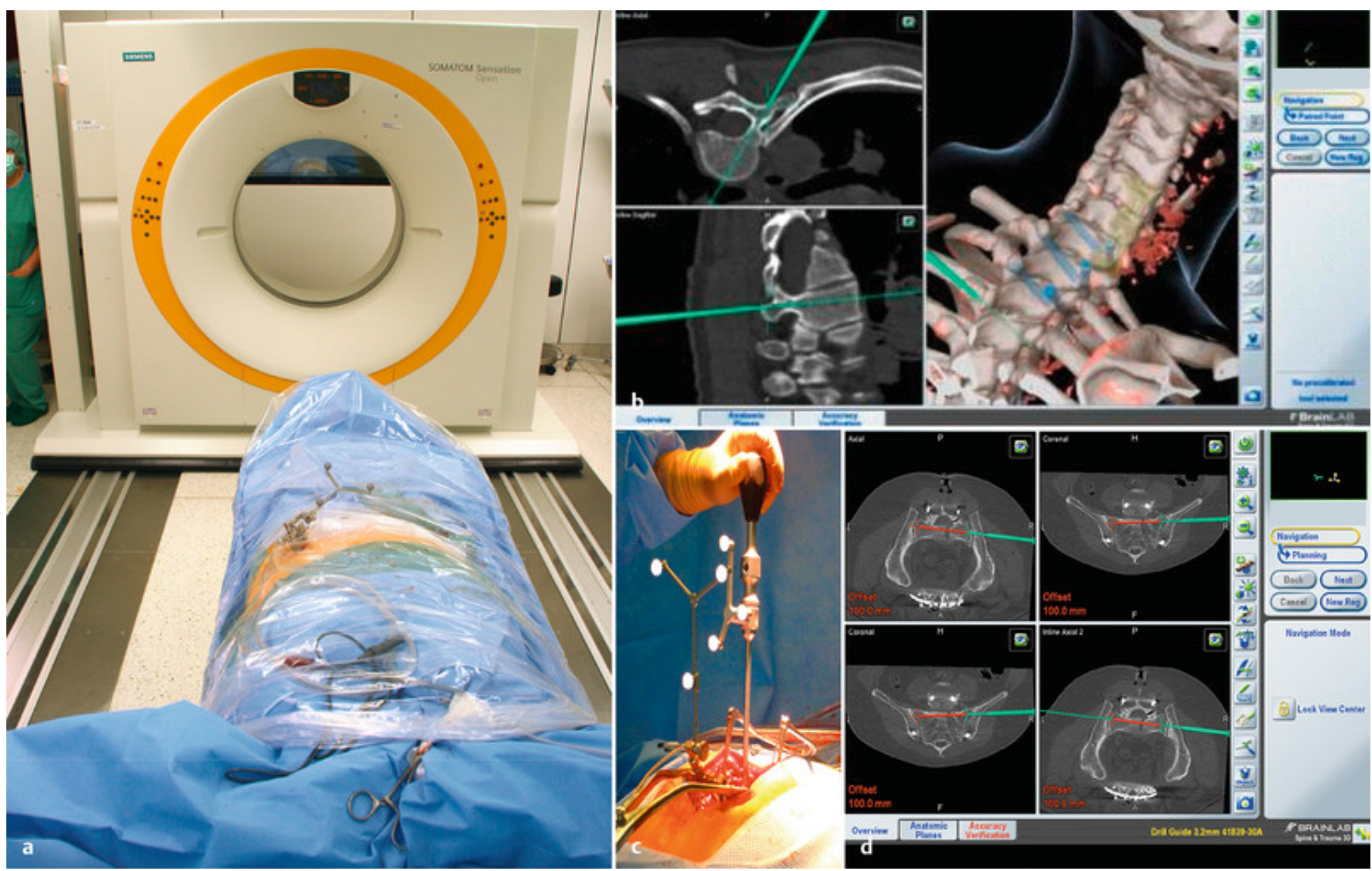

Abb. 1 a bis d a Typische CT-Geräteanordnung im OP-Saal. b, c Intraoperative Situation bei der Navigation schmaler Pedikel. Die Darstellung der Navigation kann über einen Wandmonitor oder über einen schwenkbaren und steril bedienbaren Touchscreen-Monitor erfolgen. $\mathbf{d}$ Planung einer ISG-Verschraubung.

Studien wurden zur Genauigkeit der computergestützten Navigation von Pedikelschraubenplatzierungen publiziert. In einer Metastudie, die 18 Kohortenund 2 randomisierte prospektive Studien kumulierte, zeigten Shin et al., dass die Wahrscheinlichkeit von Pedikelschraubenfehlplatzierungen im gesamten Wirbelsäulenbereich durch die Anwendung der Navigation von 15\% auf $6 \%$ gesenkt werden konnte [2]. Auch hinsichtlich der perkutanen ISG-Verschraubung zeigten sich in mehreren Studien, u. a. in einer großen Metaanalyse, Vorteile der CT-Navigation, dies nicht nur gegenüber konventionell-radiologischen Verfahren, sondern auch gegenüber der 2D- und 3D-Bildwandler gestützten Navigation $[3,4]$.

Die Genauigkeit der iCT-gestützten Navigation ist von zahlreichen Faktoren abhängig. Neben den sehr geringen software- und technologiebedingten Fehlertoleranzen können patientenspezifische Faktoren wie Adipositas oder einliegende Implantate die radiologische Qualität und damit auch die Genauigkeit erheblich beeinflussen. Aufgrund der Mobilität der Wirbelsäule nimmt die Genauigkeit der Navigation auch mit dem Ab- stand des navigierten Wirbels zum Referenzierungspunkt ab. Während die Navigation unter Verwendung eines präoperativ aquirierten CT-Datensatzes schon allein aufgrund der verschiedenen Untersuchungsbedingungen (CT in Rückenlage) größere Abweichungen erwarten lässt, bietet das iCT gerade bei langstreckigen Wirbelsäuleneingriffen hier einen Präzisionsvorteil [5]. Nicht zuletzt kommt auch dem regelmäßigen Einsatz der computergestützten Navigation eine Rolle für die Präzision zu. Rivkin et al. [6] sahen bei den ersten 30 Patienten nach Einführung des computergestützten Verfahrens eine Pedikelperforationsrate von $13,21 \%$, bei den 30 darauf folgenden Patienten lag die Perforationsrate nur noch bei $5,6 \%$ und bestätigen somit den Lernkurveneffekt.

\section{Indikationen}

In der spinalen Chirurgie wird das iCT für die Platzierung von Pedikelschrauben am häufigsten verwendet. Gerade an der HWS und im oberen BWS-Abschnitt wird - besonders bei schmalen Pedikeln - die Platzierung der Schrauben durch Anwendung der Navigation mit einem iCT erleichtert. Jeswani et al. untersuch- ten die Perforationshäufigkeit der Pedikelschrauben bei 21 Patienten mit schmalen $(<3 \mathrm{~mm})$ Pedikeln. Alle 52 Schrauben perforierten den Pedikel um nicht mehr als $1 \mathrm{~mm}$ im postoperativen CT. In keinem Fall war eine Perforation nach medial beobachtet worden [7].

Für die sichere und vollständige Resektion von Tumoren ist das intraoperative CT eine weitere sinnvolle Anwendungsmöglichkeit. Insbesondere Osteoidosteome (Fallbeispiel 1), aber auch schlecht abgrenzbare Tumore wie Chordome und Sarkome können mithilfe der CT-gestützten Navigation entfernt werden. Die intraoperative Bildgebung dient dabei der navigierten Resektion, der Darstellung etwaiger Tumorreste nach erfolgter Resektion und auch der anschließenden Navigation der Instrumentierung.

Revisionseingriffe sind häufig durch eine alterierte Anatomie der knöchernen Strukturen durch die vorangegangenen Eingriffe erschwert. Auch hier hat sich der Einsatz des iCTs bewährt [8].

Im Bereich der Unfallchirurgie hat sich die intraoperative 3D-Bildgebung v.a. bei der Versorgung von Frakturen mit 




Abb. 2a bis d Fallbeispiel 1. a Präoperatives CT mit axialer, sagittaler, koronarer Darstellung sowie 3D-Rekonstruktion des Osteoblastoms. b Präoperatives Angio-CT mit Darstellung der Lagebeziehung der rechten A. vertebralis zum Tumor. c Intraoperatives CT nach Resektion des Tumors mit Markierungsstift im Situs. d Postoperative Situation. 


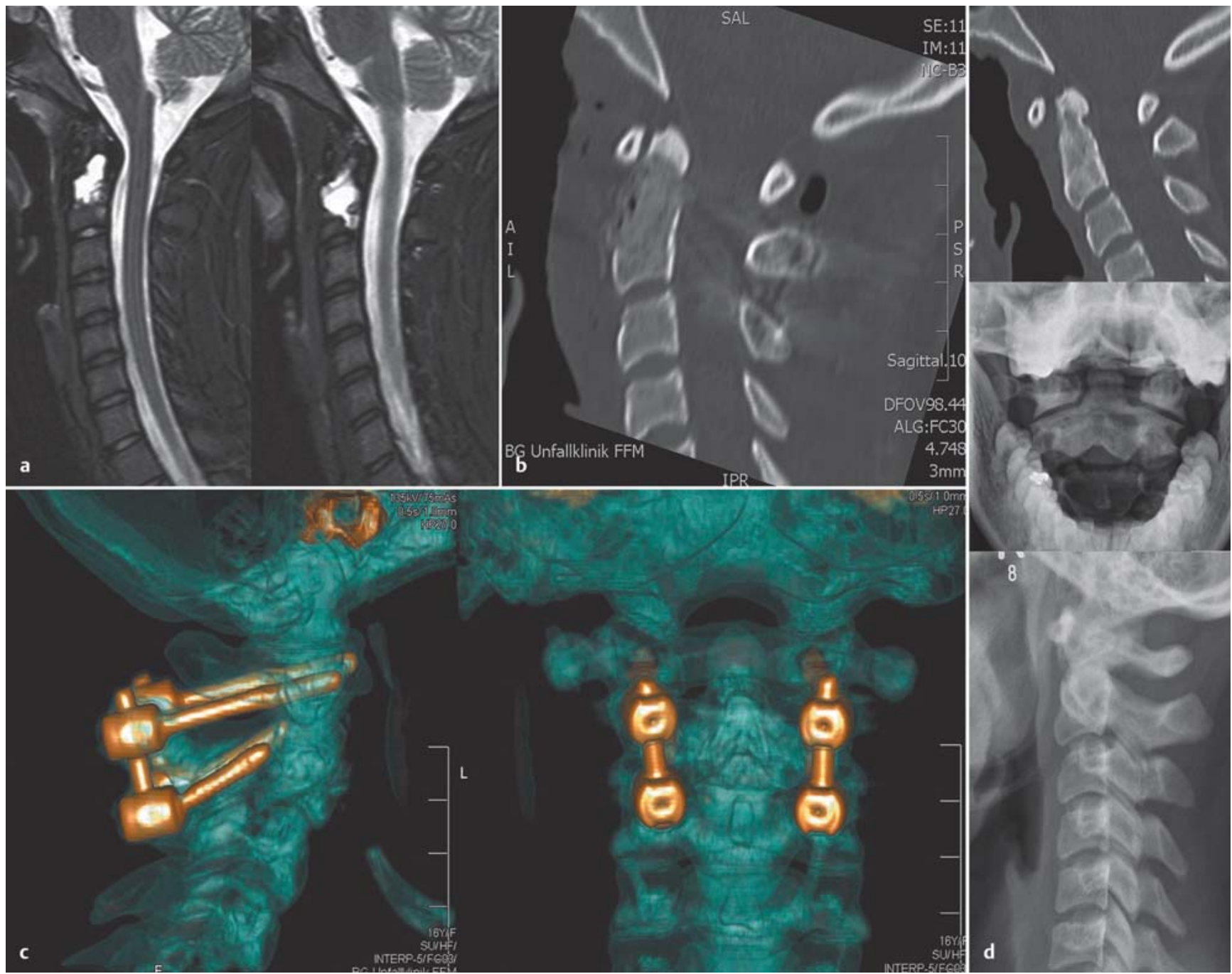

Abb. 3 a bis d Fallbeispiel 2. a Präoperatives MRT mit Darstellung einer Lyse des Dens axis. b Das intraoperative CT zeigt die vollständige Resektion und Auffüllung des Defekts mit autologer Spongiosa. c Postoperatives CT mit 3D-Rekonstruktion der Schraubenlage. d CT und Röntgendiagnostik ein Jahr nach Entfernung der Implantate.

Gelenkbeteiligung, wie bspw. Tibiakopfoder Sprunggelenks- sowie Calcaneusfrakturen, etabliert. Hier hat sich jedoch vorwiegend die 3D-Bildwandler-Technologie durchgesetzt. Die Vorteile der intraoperativen Computertomografie als reines Diagnostikum kommen erst bei großen Untersuchungsbereichen wie langstreckigen Wirbelsäulen- oder komplexen Beckeneingriffen zur Geltung, da das Field of View der fluoroskopischen Geräte in der Regel nur geringe Volumina umfasst. Hieraus erklären sich auch die Vorteile der Computernavigation auf Basis eines intraoperativen CTs.

Grundsätzlich entsprechen die sinnvollen Indikationen der CT-Navigation denen der fluoroskopischen Navigation. Neben den Pedikelschraubenplatzierungen an HWS und BWS sowie Tumorresektionen im Bereich der Wirbelsäulenchirurgie stellen v.a. die navigierte ISG-Ver- schraubung sowie ausgewählte weitere minimalinvasive Schraubenosteosynthesen, wie z.B. am vorderen und hinteren Beckenring sowie am Azetabulum, unfallchirurgische Indikationen dar. Ein Mehrwert im Vergleich zum fluoroskopischen Verfahren ergibt sich entsprechend bei multisegmentalen Wirbelsäuleneingriffen sowie multilokulären Beckeneingriffen, wie bspw. einer beidseitigen ISG-Verschraubung oder kombiniert lumbopelvinen Versorgungen.

\section{Strahlenbelastung}

Durch das iCT selbst ergibt sich keine zusätzliche Strahlenbelastung für das OPPersonal, da dieses den Kontrollbereich während der Untersuchung verlässt. Der Patient selbst ist jedoch einer Belastung im Verhältnis zum untersuchten Körperabschnitt ausgesetzt. Diese ist in der Regel höher als die übliche Strahlenbelas- tung durch intraoperative Durchleuchtung oder 3D-Fluoroskopie.

\section{Perioperative Logistik}

In unserer Klinik kommt ein auf Bodenschienen geführtes iCT-Gerät der Fa. Siemens (Somatom Sensation Open, 16-Zeiler) in Verbindung mit einem Vollcarbontisch zum Einsatz (Abb. 1). Die Steuerung des CTs erfolgt aus einem separaten Schaltraum mit Kamera-gestützter Übertragung der Narkoseüberwachung. Vor der Operation bzw. am Tagesbeginn ist eine Kalibrierung des CTs erforderlich, während der der OP-Saal aus Strahlenschutzgründen nicht betreten werden kann. Ein Vollcarbontisch ist für Anwendungen im Bereich der Brust- und Lendenwirbelsäule sowie bei pelvinen Eingriffen obligat, für die Untersuchung der Halswirbelsäule wird zumindest eine Carbon-Mayfield-Klemme benötigt. Die 




Abb. 4a bis c Fallbeispiel 3. a Präoperatives MRT mit Darstellung der Längenausdehnung des spinalen metastatischen Befalls. b Präoperative axiale CT-Schichten zeigen die Lagebeziehung der Metastasen zur Schilddrüse und den umgebenden Weichteilstrukturen und Verlagerung der Trachea nach links. c Postoperatives Ergebnis 



Abb. 5 a bis c Fallbeispiel 4. a Präoperative konventionell-radiologische, MRT- und CT-Diagnostik bei einem fünfjährigen Jungen mit JarchowLevine-Syndrom. b Intraoperative Navigation bei schmalem Pedikel. c Postoperatives Ergebnis.

Lagerung auf dem OP-Tisch bedarf im Vergleich zur konventionellen Lagerung nur weniger Modifikationen, dabei ist v.a. die Weite der Gantry-Öffnung (bei unserem Gerät $84 \mathrm{~cm}$ ) zu beachten. Dies erfordert eine Anlagerung der Arme an den Oberkörper respektive weit kranial am Narkosebügel. Dabei sind die Arme so tief zu lagern, dass selbst bei Anwendungen im BWS-Bereich eine zusätzliche C-Bogen-Darstellung bei Bedarf möglich ist. Die Patientenposition in Relation zur scanbaren Strecke wird durch einen Pro- belauf vor dem sterilen Abdecken abgeglichen. Fest-installierte, auf Schienen fahrbare CT-Scanner sind in Bezug auf die Anordnung des OP-Tisches und der Instrumentationstische nicht so flexibel wie mobile Geräte, sodass sich die Lagerung der Patienten insgesamt komplexer und fehleranfälliger darstellt.

Zur Navigation dient ein an der Decke hängendes System der Firma Brainlab (Vector Vision Sky) mit automatischer Registrierung der Patientenanatomie im
Rahmen des CT-Scans. Sowohl die Bilder des iCTs wie auch sämtliche PACS-Bilder und der Navigationsbildschirm lassen sich auf 2 hochauflösenden 50-ZollWandmonitoren in beliebiger Kombination darstellen. Die Navigation auf Basis des intraoperativen CTs kann sowohl bei offenen als auch bei minimalinvasiven Eingriffen genutzt werden. In jedem Fall ist der Referenzstern des Navigationssystems rigide an einer knöchernen Struktur in unmittelbarer Nähe zum zu navigierenden OP-Bereich anzubringen, da 


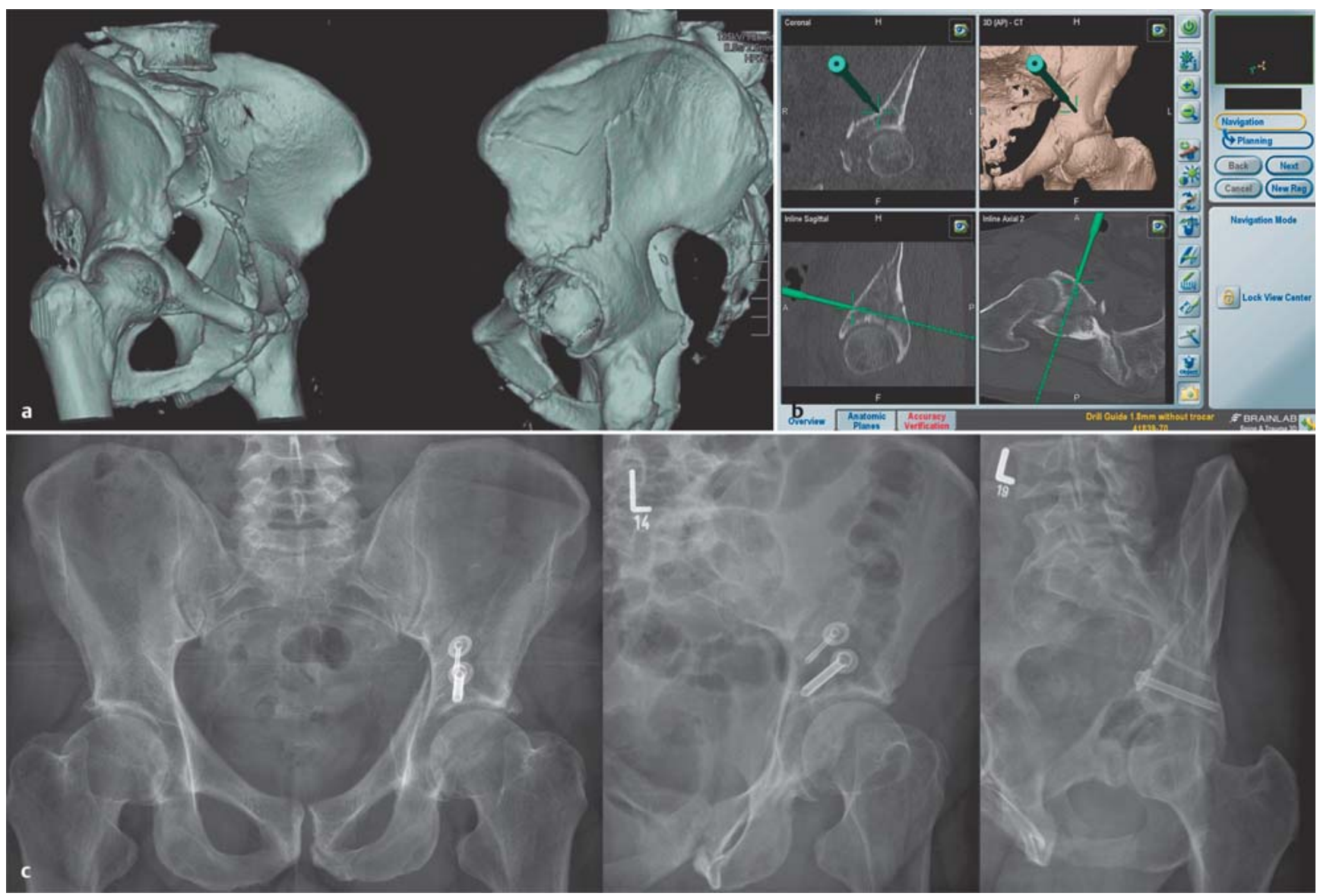

Abb.6a bis c Fallbeispiel 5. a Präoperative CT-Rekonstruktionen. b Intraoperativer Screenshot der Navigation. c Postoperative Röntgenbilder.

das Offset und damit die Genauigkeit der Navigation nicht zuletzt aufgrund der relativen Mobilität des knöchernen OP-Situs mit zunehmendem Abstand zum Referenzstern abnehmen. Die Kombination mit Befestigung an der Mayfield-Klemme ist für ventrale Eingriffe an der HWS denkbar, bislang aber nicht uneingeschränkt zu empfehlen. Eine Apnoephase während des Scans vermeidet atemabhängige Artefakte.

Die Scandauer ist vom Volumen des darzustellenden OP-Bereichs abhängig und beträgt in der Regel nur einige Sekunden. Zuvor ist jedoch noch die Anfertigung eines Topogramms erforderlich. Der zeitliche Mehraufwand im Vergleich zur bildwandlergestützten Durchleuchtung summiert sich schließlich aus der aufwendigeren Lagerung, der sterilen Abdeckung des CT-Scanners respektive des OP-Gebiets sowie der eigentlichen Untersuchung einschließlich Kalibrierung. Die Navigation erfordert zusätzlich Zeit für die Fixierung des Referenzsterns sowie die erforderlichen Software-immanenten Einstellungen und Abläufe. Demgegenüber steht die potenzielle Re- duktion der OP-Dauer eben durch Nutzung der Navigation.

\section{Kosten-Nutzen-Analyse}

Sowohl die Investitionskosten als auch die Betriebskosten für das iCT und die Navigationseinheit sind derzeit hoch. Kosten für Verbrauchsartikel wie sterile Abdeckungen, Mehrkosten durch etwaig verlängerte OP-Zeiten müssen ebenso Berücksichtigung finden wie der teilweise erhöhte Personalbedarf, da zum Betrieb eines klassischen CTs eine MTRA zugegen sein muss.

In Zeiten knapper finanzieller Ressourcen ist die Kosten-Nutzen-Analyse ein Instrument für die wirtschaftliche Effektivität des Verfahrens. Sanborn et al. verglichen die Kosten eines iCT (Medtronic O-Arm), neurophysiologischem Monitoring und intraoperativer Durchleuchtung gefolgt von postoperativer CT-Kontrolle. Sie stellten fest, dass das iCT mit den geringsten Kosten bei vergleichbarer Effektivität als Monitoringverfahren für die korrekte Pedikelschraubenlage verbunden war [9]. Andererseits schätzten
Costa et al. den Kostenvorteil des intraoperativen CTs im Vergleich zum präoperativen CT als Grundlage für die Navigation als eher gering ein [10]. Das iCT führt nicht zwangsläufig zu einer Verlängerung der Operationszeit.

Die Frage, ob die Anschaffung eines iCTSystems lohnenswert ist, lässt sich letztlich nicht pauschal mit ,ja“ oder „nein“ beantworten und hängt wesentlich vom Operationsspektrum und dem Komplexitätsgrad der Eingriffe ab. Der medizinische Vorteil liegt je nach Spektrum auf der Hand, jede Institution muss jedoch eine individuelle Analyse zur KostenNutzen-Relation vornehmen.

Vorteile der intraoperativen CT-Bildgebung sind:

- Dreidimensionale Darstellung einschließlich multiplanarer Rekonstruktionen

- Wesentlich größerer Untersuchungsbereich im Vergleich zur 2D- und 3D-Durchleuchtung

- Höchstmögliche Auflösung knöcherner Strukturen und Navigationsgenauigkeit 




Abb. 7 Fallbeispiel 6. Intraoperative Screenshots einer beidseitigen navigierten ISG-Verschraubung bei einer 67-jährige Patientin mit Insuffizienzfrakturen des Sakrums sowie des rechten Azetabulums und linken vorderen Beckenrings.

- Automatische Registrierung der Bilddaten zur Navigation

- Möglichkeit der Kontrolle des Operationsergebnisses im OP

Nachteile der intraoperativen CT-Bildgebung sind:

- Höhere Strahlenbelastung des Patienten

- Höhere Investitions-, Betriebs- und Wartungskosten

- Aufwendige Handhabung

- Keine sofortige radiologische Kontrolle wie beim C-Bogen möglich

\section{Fallbeispiele}

\section{Fallbeispiel 1}

Ein 24-jähriger Patient, der 2 Tage nach einem Verkehrsunfall über anhaltende Nackenschmerzen klagte. Das CT zeigte überraschenderweise eine tumoröse Läsion in der Pars lateralis des HWK2. Der Tumor führte zu einer partiellen Kompression und Verlagerung der A. vertebralis und konnte mithilfe der iCT-gestützten Navigation über einen dorsalen Mittellinienzugang in toto entfernt werden. Anschließend erfolgte die ventrale Fusion mittels intervertebralem Cage und Plattenosteosynthese. Histologisch handelte es sich um ein Osteoblastom (Abb. 2).

\section{Fallbeispiel 2}

Eine 16-jährige Schülerin gab nach Bodenturnen Nackenschmerzen an. Hier konnte überraschenderweise im CT eine Lyse des Dens diagnostiziert werden. Aufgrund der symptomatischen Instabilität/
Frakturgefährdung erfolgte die ventrale transorale intraossäre Resektion (histologisch: aneurysmatische Knochencyste) und Auffüllen des Defekts mit autologer Spongiosa mit anschließender temporärer atlantoaxialer Stabilisation, die unter iCT-Führung durchgeführt wurde (Abb. 3).

\section{Fallbeispiel 3}

Bei einer 19-jährigen Patientin wurde ein Schilddrüsenkarzinom szintigrafisch gesichert. Das HWS-MRT zeigte eine spinale Metastasierung per continuitatem, die die Halswirbel 5 und 6 sowie Brustwirbel 1 und 2 umfasste. Der ventralen Resektion mit Fibula-Interponat folgte die additive dorsale navigierte Instrumentation von HWK3 bis BWK7 (Abb. 4).

\section{Fallbeispiel 4}

5-jähriger Junge mit Jarcho-Levine-Syndrom. Dorsale Derotations-Korrekturspondylodese von BWK7 bis BWK12. In diesem Fall erwies sich das iCT als präzise Hilfe für die langstreckige Stabilisation bei ausgeprägter Fehlstellung (Abb. 5).

\section{Fallbeispiel 5}

65-jähriger Patient mit linksseitiger Azetabulumfraktur (vordere Pfeiler- und hintere Hemiquerfraktur) bei vorbestehender mäßiger Koxarthrose. Operative Versorgung über limitiert-offene Reposition und navigierte Schraubenosteosynthese (Abb. 6).

\section{Fallbeispiel 6}

Intraoperative Screenshots einer beidseitigen navigierten ISG-Verschraubung bei einer 67-jährigen Patientin mit Insuffizienzfrakturen des Sakrums sowie des rechten Azetabulums und linken vorderen Beckenrings (Abb. 7).

\section{Fazit}

Die intraoperative CT-Bildgebung wie auch die iCT-gestützte Navigation weisen bei speziellen Fragestellungen gegenüber der konventionellen intraoperativen 2D- und 3D-Bildgebung Vorteile auf. Der Benefit der Navigation bei der Platzierung von Pedikelschrauben konnte mehrfach, u.a. durch eine Metaanalyse, bestätigt werden. Die Strahlenbelastung ist im Vergleich zur konventionellen Durchleuchtung für den Operateur geringer, für den Patienten höher. Aufgrund der hohen Investitions- und Betriebskosten wird das Verfahren absehbar wohl nur größeren Wirbelsäulenund unfallchirurgischen Zentren vorbehalten bleiben.

\section{Literatur}

${ }^{1}$ Laine T, Lund T, Ylikoski M et al. Accuracy of pedicle screw insertion with and without computer assistance: a randomised controlled clinical study in 100 consecutive patients. Eur Spine J 2000; 9: 235-240

2 Shin BJ, James AR, Njoku IR et al. Pedicle screw navigation: a systematic review and metaanalysis of perforation risk for computernavigated versus freehand insertion. J Neurosurg Spine 2012; 17: 113-122

3 Zwingmann J, Hauschild 0 , Bode G et al. Malposition and revision rates of different imaging modalities for percutaneous iliosacral 
screw fixation following pelvic fractures: a systematic review and meta-analysis. Arch Orthop Trauma Surg 2013; 133: 1257-1265

${ }^{4}$ Hsieh JC, Drazin D, Firempong AO et al. Accuracy of intraoperative computed tomography image-guided surgery in placing pedicle and pelvic screws for primary versus revision spine surgery. Neurosurg Focus 2014; 36: E2

5 Costa F, Cardia A, Ortolina A et al. Spinal navigation: standard preoperative versus intraoperative computed tomography data set aquisition for computer-guidance system: radiological and clinical study in 100 consecutive patients. Spine 2011; 36: 2094-2098

${ }^{6}$ Rivkin MA, Yocon SS. Thoracolumbar instrumentation with CT-guided (O-Arm) navigation in 270 consecutive patients: accuracy rates and lessons learned. Neurosurg Focus 2014; 36: E7
${ }^{7}$ Jeswani S, Darzin D, Hsieh JC et al. Instrumenting the small thoracic pedicle: the role of intraoperative computed tomography imageguided surgery. Neurosurg Focus 2014; 36: E6

${ }^{8}$ Hsieh JC, Darzin D, Firempong AO et al. Accuracy of intraoperative computed tomography image-guided surgery in placing pedicle and pelvic screws for primary versus revision spine surgery. Neurosurg Focus 2014; 36: E2

${ }^{9}$ Sanborn MR, Thawani JP, Whitmore RG et al. Cost-effectiveness of confirmatory techniques for the placement of lumbar pedicle screws. Neurosurg Focus 2012; 33: E12

10 Costa F, Porazzi E, Restelli $U$ et al. Economic study: a cost-effectiveness analysis of an intraoperative compared with a preoperative image-guided system in lumbar pedicle screw fixation in patients with degenerative spondylolisthesis. Spine J 2014; 14: 1790-1796
Dr. med. Christoph-H. Hoffmann

Oberarzt

Zentrum für Wirbelsäulenchirurgie und Neurotraumatologie Dr. med. Philipp Wilde Oberarzt

Prof. Dr. med. Reinhard Hoffmann Ärztlicher Direktor

Klinik für Unfallchirurgie und Orthopädische Chirurgie Prof. Dr. med. Frank Kandziora Chefarzt

Zentrum für Wirbelsäulenchirurgie und Neurotraumatologie BGU Frankfurt am Main Friedberger Landstraße 430 60389 Frankfurt

ws@bgu-frankfurt.de 